\title{
A Metamodel for the Measurement of Object-Oriented Systems: An Analysis using Alloy
}

\author{
Jacqueline A. McQuillan \\ Department of Computer Science \\ National University of Ireland Maynooth \\ Co. Kildare, Ireland \\ jmcq@cs.nuim.ie
}

\author{
James F. Power \\ Department of Computer Science \\ National University of Ireland Maynooth \\ Co. Kildare, Ireland \\ jpower@cs.nuim.ie
}

\begin{abstract}
This paper presents a MOF-compliant metamodel for calculating software metrics and demonstrates how it is used to generate a metrics tool that calculates coupling and cohesion metrics. We also describe a systematic approach to the analysis of MOF-compliant metamodels and illustrate the approach using the presented metamodel. In this approach, we express the metamodel using UML and $O C L$ and harness existing automated tools in a framework that generates a Java implementation and an Alloy specifcation of the metamodel, and use this both to examine the metamodel constraints, and to generate instantiations of the metamodel. Moreover, we describe how the approach can be used to generate test data for any software based on a MOF-compliant metamodel. We extend our framework to support this approach and use it to generate a test suite for the metrics calculation tool that is based on our metamodel.
\end{abstract}

\section{Introduction}

Software metrics are important in many areas of software engineering, for example assessing software quality or estimating the cost and effort of developing software. Many metrics have been proposed and new metrics continue to appear in the literature regularly [9]. Many of these metrics are incomplete, ambiguous and open to a variety of different interpretations [3]. This makes it difficult to create general metric tools and everytime a new metric is defined the tools need to be updated with the new metric [18]. Furthermore, many of these metrics are applicable to a number of different models of a software system. In order to provide assurance that the same concepts are being measured from these different models we need a way to specify the metrics in a generic way, independent of the particular model.

Like Mens and Lanza, we believe that these issues are best addressed using a language-independent, metricsspecific metamodel [18]. However, they do not consider coupling or cohesion metrics in their work. In this paper we present a metamodel for calculating object-oriented software metrics which is based on existing frameworks for coupling and cohesion measurement $[3,4]$. We use the metamodel to specify a set of existing coupling and cohesion metrics and use our existing Eclipse-based metrics framework [15] to automatically generate a tool to calculate these metrics.

Developing and working with metamodels can be difficult since they deal with abstract concepts. Therefore, it is important that we are able to perform analysis on metamodels and assess their correctness. By correctness, we mean that the metamodel specification is consistent and adequately describes what the user intends. Also, in order to ensure the correctness and quality of software applications that are based around metamodels, for example our metrics tool, we need to be able to test these applications. However, there is no direct way of automatically generating instantiations of a metamodel to use as test inputs for testing metamodel-based applications [7].

In this paper we describe an approach to the analysis of Meta Object Facility (MOF)-compliant metamodels and apply it to our metrics specific metamodel. In our approach, we express the metamodel using the Unified Modelling Language (UML) and the Object Constraint Language (OCL) [22], and harness existing automated tools in a framework that generates a Java implementation of our metamodel.

We also generate an Alloy [12] specification corresponding to the metamodel, and use this to examine the metamodel constraints and to generate sample instances of the metamodel. Our reflective instantiator takes these Alloy generated models and transforms them into instances of the Java implementation, thus harnessing Alloy's lightweight approach to generate a test suite for our metrics tool. We 
use this test suite to determine if the tool correctly computes metric values for the coupling and cohesion metrics. Finally, we evaluate the adequacy of the generated test suite in terms of traditional line and branch coverage criteria.

This paper is organized as follows. Section 2 outlines some of the background information. Details of our metrics specific metamodel are presented in Section 3. In Sections 4 and 5, we describe an approach to the analysis of MOFcompliant metamodels and illustrate the approach using our metamodel. In Section 6, we describe the generation of a test suite for the metamodel-based metrics tool. Section 7 presents a discussion of related work. Section 8 summarises and concludes the paper.

\section{Background}

Models provide a representation of a real system and are increasingly important in software engineering, particularly in the Model Driven Engineering (MDE) approach [22]. Typically, we think of a model of a software system as being a design model, such as UML class or sequence diagrams, or an implementation model, such as an actual program. As the name suggests, a metamodel is a model that is used to describe the structure of other models. One example of a metamodel specification is the UML Superstructure Specification from the Object Management Group (OMG) [21], which defines models for each of the diagrams of the UML.

While a number of different formalisms may be used to describe metamodels, one of the most widely adopted standards is the Meta Object Facility (MOF), which is specified by the OMG [20]. The MOF provides a set of constructs for defining metamodels and is referred to as a metametamodel. As well as facilitating comprehension, using a standard metamodelling formalism aides interoperability, through formats such as the XML Metadata Interchange (XMI), as well as automated tool generation.

\subsection{Metamodels and metrics}

Many software metrics have been proposed in the literature $[6,9]$. For these software metric definitions to be usable, it is important that the definitions clearly specify what is to be counted. For example, when counting method calls, do we include calls to abstract methods, calls to/from inherited and overridden methods etc. Briand et al. have shown that even seemingly straightforward metric definitions are subject to a range of different interpretations [3, 4].

Several authors have considered the use of metamodels as a way to address the problem of ambiguous metric definitions. Such an example is the canonical presentation of coupling and cohesion metrics by Briand et al. which was effectively based around a metrics specific metamodel of an object-oriented software system. Mens and Lanza [18] propose a language independent metamodel for object-oriented metrics that is based on graphs. They use this to define a selection of generic object-oriented metrics and higher order metrics but do not consider coupling or cohesion metrics.

Recent research has built upon this work by defining metrics as queries over metamodels. El-Wakil et al. propose the use of XQuery as a metric definition language to extract metric data from XMI documents, specifically UML designs [8]. Harmer and Wilkie, working from a relational schema, express metric definitions as SQL queries over this schema [23]. Baroni et al. propose using the OCL and the UML 1.3 metamodel to define UML-based metrics [2].

In our own work, we have extended the approach of Baroni et al. in a manner specifically designed to be reusable for other metamodels [15]. We have used the Dagstuhl Middle Metamodel as a general programming metamodel, and have defined several object-oriented metrics across this metamodel using OCL [14]. We have also defined similar metrics at the design level using the UML 2.0 metamodel [15].

However, each of these approaches is either metamodelspecific (e.g. the UML metamodel), or uses a metrics specific metamodel, with the associated difficulty of finding model instances to use as test data. The approach presented in this paper addresses this issue by providing a means of generating suitable instances to use for testing any software based on a metrics specific (or any MOF-compliant) metamodel.

\subsection{The Alloy language and analyser}

Alloy is a formal specification language based on typed first-order relational logic [12]. It has been used primarily to explore abstract software models and to assist in finding and correcting flaws in these models. An Alloy specification is based around signatures and formulas. Signatures are used for defining the entities of the model and consist of a set of declarations that define the relations and operations of the entity. Formulas such as facts, predicates and assertions are used to specify constraints on the model.

A fully automatic tool, called the Alloy Analyser has been developed simultaneously with the Alloy language. This is a "model-finder" tool that uses a constraint solver to analyse models written in Alloy. There are two types of analysis offered by the tool, namely simulation and checking. Simulation involves finding model instances that meet the Alloy specification. Checking involves finding counterexamples to the specification. To make instance finding feasible, a user may specify a scope for the model under analysis. The scope puts a bound on how many instances of an entity may be observed in a model instance and thus limits the number of model instances to be examined. 


\section{A metamodel for object-oriented software measurement}

In this section we describe the motivation for developing our metamodel for coupling and cohesion measurement. We also present the details of the metamodel and with the use of an example illustrate how it is used in the definition and calculation of software metrics.

\subsection{Motivation}

Typically, calculating software metrics involves counting entities found in a model, such as number of classes or methods [9]. While some metrics are specific to a given type of model (e.g. lines of code), others are applicable to a number of different models. For example, coupling between classes might be usefully measured in source code, in a UML class diagram, or even a UML sequence diagram.

If similar metrics are defined on different models of the same system, then it is desirable that we can make these similarities explicit. For example, we should be able to ensure that the rules for calculating the depth of an inheritance tree are consistent between the source code and the corresponding UML class diagram. Ideally, it should be possible to define a set of metrics once, and then adapt them to each relevant model in turn. It is our view that this is best addressed by defining the metrics as OCL queries over a metrics specific metamodel and mapping all other metamodels to this canonical metamodel [16].

The advantages of a such an approach include:

- the ability to specify standardised, unambiguous definitions for software metrics.

- providing assurance that the same concepts are being measured from the different models of the same software system.

- the automation of the generation of a measurement tool by forward engineering the metamodel and OCL definitions to a language implementation.

\subsection{Metamodel overview}

One requirement of our metamodel is that it is interoperable with the UML and Java metamodels and thus has been developed to conform to the MOF. This ensures that all three metamodels are specified using the same formalism, thus facilitating the translation of instances of the UML and Java metamodel to instances of the metamodel presented in this paper.

Moreover, our MOF-compliant metamodel is based on the coupling and cohesion measurement frameworks proposed by Briand et al. [3, 4]. It captures the basic structure of an object-oriented system at a level of abstraction that
Table 1. Summary of implemented metrics.

\begin{tabular}{ll}
\hline Metric Set & Metrics from references $[6, \mathbf{6}, \mathbf{4}]$ \\
\hline CKMetrics & WMC, NOC, DIT \\
Cohesion & LCOM1, LCOM2, LCOM3, LCOM4, LCOM5, \\
& Co, NewCo, TCC, LCC, ICH \\
Coupling & RFC, RFC', CBO, CBO', MPC, COF, DAC, DAC', \\
& ICP, IH_ICP, NIH_ICP, IFCAIC, ACAIC, OCAIC, \\
& ACMIC, OCMIC, IFCMIC, AMMIC, OMMIC, \\
& IFMMIC, FMMEC, DMMEC, OMMEC, FCMEC, \\
& DCMEC, OCMEC, OCAEC, FCAEC, DCAEC
\end{tabular}

represents concepts and relationships required for coupling and cohesion measurement.

The metamodel is composed of a single package called $M M$ (Metrics Metamodel) and the contents of this package are depicted in Figure 1. The figure shows the main classes involved in the metamodel, along with the important associations, necessary for distinguishing the different types of coupling and cohesion metrics. The central classes for coupling and cohesion metrics are Class, Method and Attribute. A Class is generalised by Type, which also generalises built-in types (e.g integer, string) and user-defined types (e.g struct, enumeration).

In order to implement the metric definitions, we distinguish between declared/implemented attributes and methods based on whether they physically appear in the class definition, or whether they are just present due to inheritance. Similarly, we partition methods into three types: those that are inherited without change, those that are inherited and overridden, and those that are declared for the first time in a class. Other classifications of methods are given by the attributes of the Method class.

Our MOF-compliant metamodel specification also contains constraints, which specify semantic and syntactic properties of the data described by the metamodel. These constraints are specified using the OCL and referred to as well-formedness rules. In total, the metamodel contains 15 well-formedness rules. Further details of these rules are given in Section 5 and a complete specification of them along with a more detailed description of the metamodel can be found in [17].

\subsection{Defining metrics using the metamodel}

In this section we describe how we used our metamodel to define three sets of existing object-oriented software metrics. The three sets of metrics CKMetrics, Cohesion and Coupling were taken from $[6,3,4]$, respectively. In total, we defined 42 metrics and these are summarised in Table 1.

In keeping with the approach outlined in [15], the metrics were defined as OCL queries over the metamodel. The 


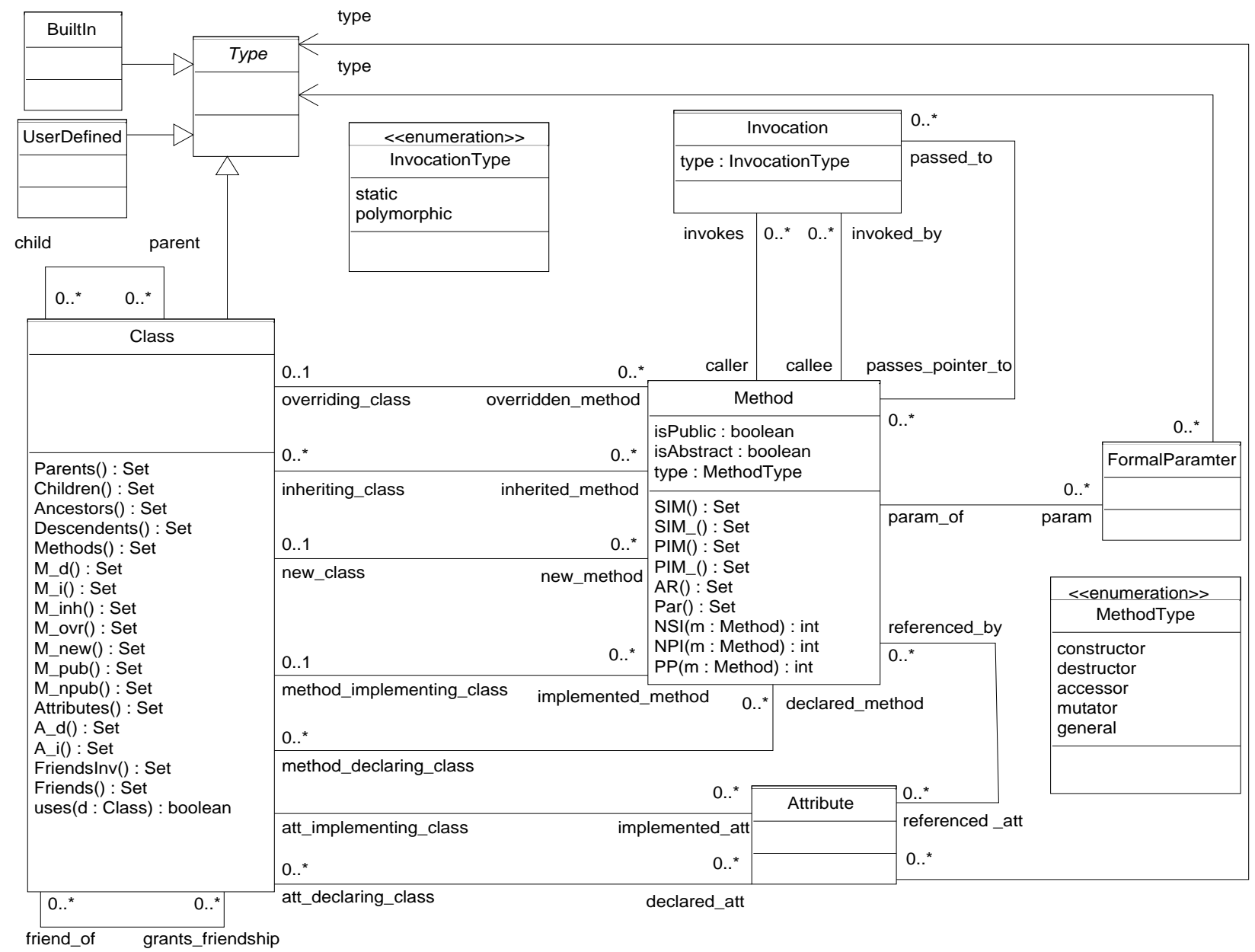

Figure 1. A class diagram showing the main classes used in the metamodel.

metamodel was extended with a separate metrics package containing a single class called Metrics, and each set of metrics was defined as follows:

1. A class was created in the metrics package for the metric set; this class extends the Metrics class.

2. For each metric, an operation was declared in the class, parameterised by the appropriate metamodel elements.

3. The metrics were defined by expressing them as OCL queries using the OCL body expression.

As an example of a definition, Figure 2 presents the definition of the number of children (NOC) metric. Here, the definition is parameterised by a single Class, and the body of the definition returns the size of the set of all children of this class. The auxiliary operation Children defined in the metamodel traverses the elements and relationships in the metamodel to assemble this set. Full details of this and the other metric definitions can be found in [17].

We have developed a measurement framework for the definition and calculation of software metrics based around an Eclipse plug-in [15]. This framework was used to define all 42 metrics shown in Table 1 and to automatically create a tool to calculate these metrics. The metrics calculation tool was created by transforming the OCL and UML corresponding to the metric sets to Java code. In brief, the tool computes the metric values for metamodel instances by invoking the Java methods corresponding to the OCL metric definitions. In Section 6, we report on how we evaluate the correctness of this metrics tool.

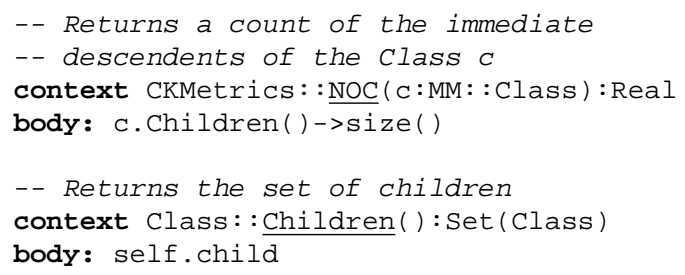

Figure 2. Definition of the $N O C$ metric in OCL using our metamodel. 


\section{An overview of the approach}

In this section we present an overview of an approach that can be used to both analyse a MOF-compliant metamodel and to automatically generate test data for software based on the metamodel.

An overview of the approach is depicted in Figure 3. In this figure, our system is delineated by a dashed red line. The inputs to the system are the metamodel and its constraints expressed as UML and OCL, and are shown on the left of the figure. The outputs of the system are shown on the bottom, and consist of a Java implementation of the metamodel and its associated OCL constraints and queries, along with a test suite based on the metamodel. These are linked through a coverage analysis, as described in Section 6 .

Both Octopus ${ }^{1}$ and Alloy are third-party tools used in our system. The UML2Alloy tool used here is a reimplementation of the same tool of Anastasakis et al. [1], but specialised for Octopus. The Reflective Instantiator tool was developed by us. The process is almost fully automated, with user intervention limited to providing the original UML/OCL description of the metamodel, and examining the generated Alloy specification. This is depicted by the stick-figure in green in Figure 3.

There are six main steps in this process:

Step 1: Expressing the metamodel in UML and OCL. The OMG specification for MOF does not define a textual or graphical representation for MOF [20]. However, there is a UML Profile that defines a bi-directional mapping between UML and MOF. The profile facilitates the creation of metamodels using UML and the viewing of MOF metamodels. Our approach uses this to express the metamodel in UML and OCL. For example, any constraints on the MOF metamodel map directly to UML constraints. This process was not automated, the metamodel was depicted using a standard UML modelling tool. Octopus is used to check the OCL for correct syntax and use of metamodel elements.

Step 2: Generating a Java implementation of the metamodel. After the metamodel and its constraints are depicted using UML/OCL, Octopus is used to generate the corresponding Java classes. All attributes and associations in the metamodel are created as fields in the appropriate classes. Finally, methods are created that check the constraints and multiplicities of the model.

Step 3: Transforming the metamodel to Alloy. We have created a tool to convert an Octopus UML/OCL metamodel to Alloy. Since this tool mimics the UML2Alloy tool [1], we only briefly outline the transformation approach here.

${ }^{1}$ http://www.klasse.nl/octopus

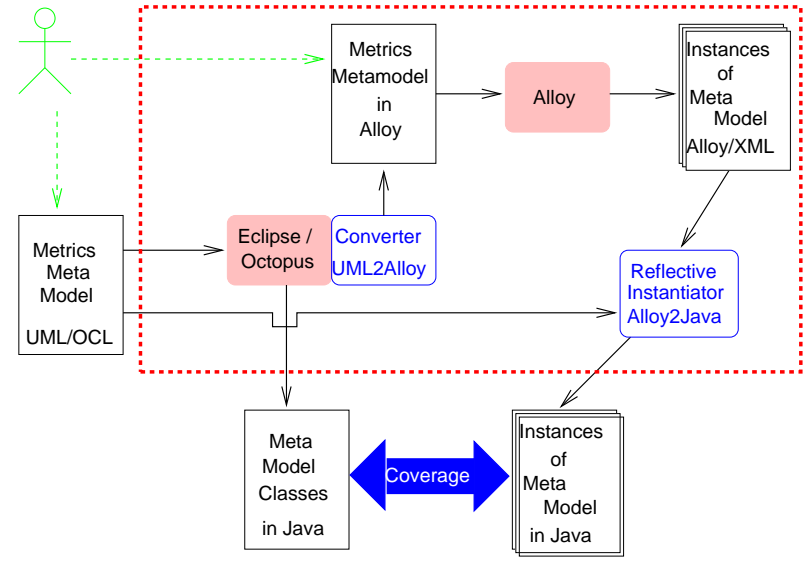

Figure 3. Overview of the approach to analysing the MOF-compliant metamodel.

Classes and Enumerations map to Alloy signatures. All attributes map to fields of the corresponding Alloy signature. Associations are also mapped to fields in the appropriate Alloy signatures. An additional fact is generated in the Alloy specification for bi-directional UML associations to show that the relations are symmetric.

Finally, any OCL invariants of the metamodel are mapped directly to Alloy facts. At present, the OCL map does not cover the full language, and requires some user intervention for more difficult constructs.

Step 4: Analysis of the metamodel. The Alloy Analyser is used to analyse the Alloy model to detect flaws in the metamodel specification. For example, it can be used to generate random instances of the metamodel that conform to the well-formedness rules. If an instance cannot be found then there is an inconsistency in the metamodel specification. It is also possible to enumerate and explore all possible instances of the metamodel. This is useful to identify invalid instances i.e. instances that do not represent what the user intends their specification to represent.

Step 5: Generation of metamodel instances using Alloy. The role of Alloy in our system is twofold. First, it allows us to investigate the metamodel constraints to check for redundancies or errors (see Section 5). Second, it allows us to automatically generate valid metamodel instances.

For this step we created a Java program to harness Alloy's model generation capabilities. This program reads an Alloy specification file and continually creates instances of the metamodel until all possible instances have been generated. Every metamodel instance produced during this step is output and stored in XML format for future use. 
Step 6: Transformation of metamodel instances to Java objects. One of the central technical contributions of our system is the Reflective Instantiator, which transforms the XML versions of Alloy-generated models into instances of the Java implementation of our metamodel.

The Reflective Instantiator parses the XML produced by Alloy and creates instances of our metamodel using the class files generated in Step 2. It does this using Java reflection, reading the class names from the XML files and creating instances of these classes. The fields of these classes are set by reading the fields from the XML and calling the appropriate set methods.

It is important to note that this process is not tied to any specific metamodel. Since the Alloy model and Java metamodel implementation are generated from the same MOF metamodel, Java reflection can make the link between them without having this information statically hard-coded. Therefore, this program is not specific to the metamodel under consideration and can be used for any metamodel.

\section{Metamodel development and analysis}

While the approach outlined in Section 4 will work for any MOF-compliant metamodel, our original intention was the specification and analysis of a metamodel for coupling and cohesion measurement. In this section we elucidate our approach using that metamodel.

\subsection{Applying the approach}

As described in Section 4, the first step of our approach is to express the metamodel in UML and OCL. As we were basing our metamodel on that of Briand et al., we began by expressing the concepts described in $[3,4]$ as a class diagram and formalised any well-formedness rules that were expressed in natural language by Briand et al.. An example of such a rule is that the set of all new, overriding and inherited methods of a class are disjoint. We suspected that all these constraints were not sufficient to describe our metamodel and thus added 15 more constraints, resulting in a total of 27 well-formedness rules. Once we had formalised all of the rules in OCL, we used Octopus to statically check the OCL constraints and then translated the MOF-compliant metamodel and its well-formedness rules to Alloy.

An example of the translation of UML classes to Alloy is shown in Figure 4. This figure gives the Alloy specification for the Class element of our metamodel which is defined in Alloy as a signature extending the Type signature. The associations for a class are represented by fields, which we have shown here in four groups. These groups represent inheritance relationships, friendship relationships (for $\mathrm{C}++$ ), and an association with the class' attributes and methods.

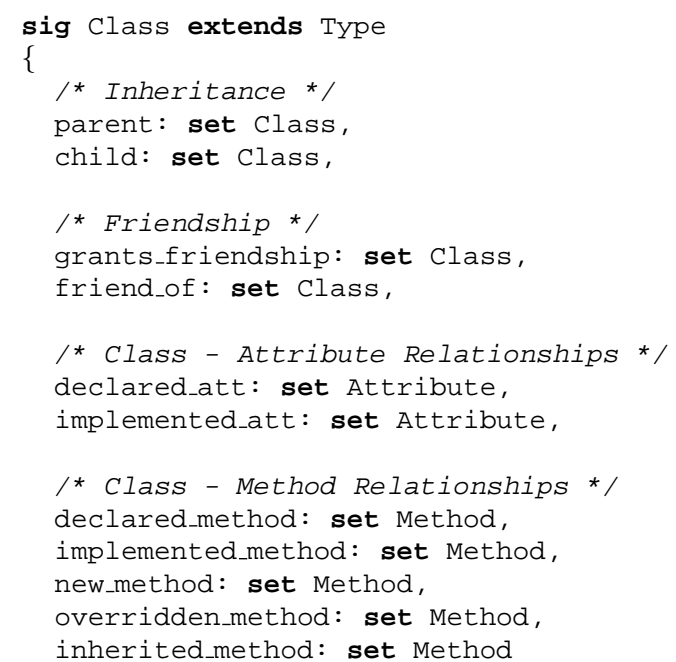

Figure 4. Alloy signature for the element Class of the metamodel.

\subsection{Metamodel analysis}

To perform the analysis, the Alloy Analyser was used to generate a random instance of the metamodel. The Analyser requires that a scope is specified for the model and then performs the analysis by exhaustively searching the state space for this scope. We specified a scope of 10 for all elements. The analyser searches for a model that contains at most 10 instances of each base class of the metamodel and conforms to the well-formedness rules of the metamodel. An instance was produced thus demonstrating that the well-formedness rules specified for the metamodel were consistent.

We then used the Analyser to search for invalid instances of the metamodel. We specified a scope of 1 for the Alloy model and manually inspected the random instances produced by the Analyser. Each time an invalid instance was found, we added a constraint to prevent that instance from being generated. For example, we found a metamodel instance where a class could inherit from itself. On completion we had a total of 37 metamodel constraints.

Upon visual inspection of the 37 metamodel constraints, we suspected that a number of the constraints were superfluous. For each of these constraints, we converted it into an assertion about the metamodel and then used Alloy to check whether the assertion was valid. If the assertion produced a counterexample then we knew that the constraint was required. If a counterexample could not be found within a reasonable scope then it cannot be guaranteed that the constraint is redundant but it can increase our confidence that it is. Therefore, we assumed that the constraint was super- 
fluous and omitted it from the specification. During this final analysis, 24 constraints were identified as potentially redundant and removed from the Alloy specification. We also found that a further 2 constraints were needed to prevent invalid metamodel instances, thus giving us a total of 15 constraints in the Alloy version of the metamodel.

\subsection{Discussion}

This approach relies on Jackson's small scope hypothesis, which suggests that if a bug exists it will appear in fairly small models of a system [12]. So, it is possible our approach may not be applicable to larger metamodels. However, in such a situation it may be possible to apply the approach by partitioning and abstracting the metamodel into the parts that are related to the properties being analysed.

Moreover, we are fully aware that this process is not a completely formalised method for developing and analysing metamodels. However, we believe that this approach gives the developer a formal way of analysing and checking for any suspected deficiencies in their metamodel specification. By iteratively analysing and improving the metamodel, the developer becomes more confident in their specification.

Finally, it is important to note that this approach is not specific to a particular metamodel. It is generally applicable to any MOF-compliant metamodel. In fact, the approach is not restricted to metamodels but is applicable to any kind of model, for example a UML class diagram of a UML model.

\section{Test suite generation}

As described in Sections 3 and 4 we were able to automatically generate both an implementation of the metamodel and an implementation to calculate the specified metrics. In this section we describe the final step in integrating the use of Alloy with this code: the construction of a test suite for the automatically generated metrics tool. We use this test suite as input to the metrics tool and use a test oracle to determine whether or not the metric results produced by the tool are correct. The test oracle had to be constructed manually and therefore, required a test suite with the following properties:

1. Each test case should contain a relatively small number of elements.

2. The number of test cases in the test suite should also be relatively small.

3. The test suite should provide as much coverage of the implementation as possible.

\subsection{Test case generation}

Using our reflective instantiator described in Section 4 we were able to automate the generation of a set of test cases
Table 2. Groups of generated test cases.

\begin{tabular}{clcc}
\hline $\begin{array}{c}\text { Test } \\
\text { Group }\end{array}$ & Alloy Command & \multicolumn{3}{c}{$\begin{array}{c}\text { No. of } \\
\text { Test Cases }\end{array}$} \\
\hline 1 & $\begin{array}{l}\text { run show for exactly 1 Type, } \\
\text { exactly 1 Attribute, exactly 1 } \\
\text { Method, exactly 1 FormalParame- }\end{array}$ \\
& $\begin{array}{c}\text { ter, exactly 1 Invocation } \\
\text { run show for 1 }\end{array}$ \\
\hline 3 & run show for exactly 1 ... all \\
& classes listed & 360 \\
\hline 4 & run show for exactly 2 Type, \\
& exactly 2 Attribute, exactly 2 \\
& Method, exactly 2 FormalParame- \\
& ter, exactly 2 Invocation \\
\hline
\end{tabular}

for the metrics calculation tool. As we required models with a relatively small number of elements we began by generating models using a small scope. Table 2 summarises the results of generating these test cases which are partitioned into four different groups:

Group 1 consisted of all possible instances with exactly one instance of each base class in our metamodel.

Group 2 is all possible instances where each base class is observed 0 or 1 times in a metamodel instance.

Group 3 is similar to group 1 except that we defined a scope of exactly 1 for all classes (not just base classes).

Group 4 again is similar to group 1 except that we allowed a scope of exactly 2 for all base classes.

\subsection{Test cases and expected results}

We added the two extra constraints to the original UML/OCL specification, and thus the generated Java implementation contained 39 constraints in total. All of the test cases summarised in Table 2 were used as input to our Reflective Instantiator. For each model, the Instantiator built the instantiation, ran the code to check each of the 39 OCL constraints, and then systematically tore down each model to test the element removal code. As each test model was built it was used as input to the metrics calculation tool and the values for all 42 metrics were recorded.

Since each generated constraint was checked for each test case, this provided further assurance that the reduced set of constraints used to generate the Alloy models was sufficient. Further, using such a large number of test cases demonstrates the robustness of the metric calculation tool and was used as a smoke test to ensure that the recorded values were within reasonable boundaries. Based on the scope used to generate each of the groups in Table 2 we computed the maximum and minimum values possible for each of the metrics. We then identified the models that produced metric values outside of these bounds. The results of this smoke test are discussed later in this section. 
Table 3. Line/Branch coverage excluded from the coverage targets.

\begin{tabular}{lcc}
\hline Reason for exclusion & Line & Branch \\
\hline Negative test cases & $11 \%$ & $1 \%$ \\
Field setters & $6 \%$ & $2 \%$ \\
Passed-as-Pointer Association & $3 \%$ & $4 \%$ \\
\hline Total excluded & $20 \%$ & $7 \%$ \\
\hline
\end{tabular}

Table 4. A breakdown of the metamodel coverage for each of the test groups in Table 2.

\begin{tabular}{c|ccc|ccc}
\hline Test & \multicolumn{2}{|c|}{ Cum. Line Coverage } & \multicolumn{3}{c}{ Cum. Branch Coverage } \\
Group & MM & Metrics & All & MM & Metrics & All \\
\hline 1 & $44 \%$ & $68 \%$ & $51 \%$ & $55 \%$ & $60 \%$ & $57 \%$ \\
2 & $49 \%$ & $68 \%$ & $54 \%$ & $62 \%$ & $60 \%$ & $61 \%$ \\
3 & $49 \%$ & $68 \%$ & $54 \%$ & $62 \%$ & $60 \%$ & $61 \%$ \\
4 & $71 \%$ & $99 \%$ & $79 \%$ & $91 \%$ & $99 \%$ & $93 \%$ \\
\hline
\end{tabular}

Our original intention was to generate a test suite with a relatively small number of test cases whose metric values could be calculated manually, serving as a test oracle for the generated metric tool. However, since the number of test cases produced is in excess of 500,000, it is necessary to reduce this suite to a more manageable size. We decided to measure the coverage of the implementation in terms of traditional code coverage criteria and to reduce the number of test cases based on these criteria.

\subsection{Coverage analysis}

Cobertura $^{2}$ was used to measure the line and branch coverage of the metamodel implementation and the implementation corresponding to the metrics. Cobertura is a free Java tool that computes the percentage of code accessed by tests.

It was not possible to achieve full line and branch coverage of the implementation for several reasons, summarised in Table 3. Since our test suite only included positive test cases, code that involves catching exceptions when the invariants of the metamodel are violated was not fully covered. Some auxiliary routines, such as alternative set and get methods were not called in constructing the model. For simplicity, the part of the metamodel dealing with method pointers was not instantiated in Alloy, significantly reducing the number of models created. Thus, excluding these totals, from our target coverage gave a maximum possible coverage of $80 \%$ for line and $93 \%$ for branch coverage.

The results of the coverage analysis is summarised in Table 4 on a per-group basis. This table has one row for each of the test case groups described previously in Table

\footnotetext{
${ }^{2}$ http: //www. cobertura.sourceforge.net/
}

Table 5. Test cases in the reduced test suite.

\begin{tabular}{c|ccc|ccc}
\hline Test & \multicolumn{2}{|c}{ Cum. Line Coverage } & \multicolumn{2}{c}{ Cum. Branch Coverage } \\
Case & MM & Metrics & All & MM & Metrics & All \\
\hline T1 & $43 \%$ & $66 \%$ & $50 \%$ & $55 \%$ & $59 \%$ & $56 \%$ \\
T2 & $44 \%$ & $68 \%$ & $51 \%$ & $55 \%$ & $60 \%$ & $57 \%$ \\
T3 & $44 \%$ & $68 \%$ & $51 \%$ & $55 \%$ & $60 \%$ & $57 \%$ \\
T4 & $44 \%$ & $68 \%$ & $51 \%$ & $56 \%$ & $60 \%$ & $57 \%$ \\
T5 & $48 \%$ & $68 \%$ & $54 \%$ & $62 \%$ & $60 \%$ & $61 \%$ \\
T6 & $59 \%$ & $68 \%$ & $54 \%$ & $62 \%$ & $60 \%$ & $61 \%$ \\
T7 & $63 \%$ & $88 \%$ & $71 \%$ & $80 \%$ & $88 \%$ & $83 \%$ \\
T8 & $68 \%$ & $89 \%$ & $74 \%$ & $87 \%$ & $89 \%$ & $87 \%$ \\
T9 & $68 \%$ & $89 \%$ & $74 \%$ & $87 \%$ & $89 \%$ & $88 \%$ \\
T10 & $68 \%$ & $89 \%$ & $74 \%$ & $87 \%$ & $89 \%$ & $88 \%$ \\
T11 & $69 \%$ & $97 \%$ & $77 \%$ & $87 \%$ & $98 \%$ & $90 \%$ \\
T12 & $69 \%$ & $97 \%$ & $77 \%$ & $87 \%$ & $98 \%$ & $90 \%$ \\
T13 & $69 \%$ & $98 \%$ & $77 \%$ & $87 \%$ & $99 \%$ & $91 \%$ \\
T14 & $71 \%$ & $99 \%$ & $79 \%$ & $91 \%$ & $99 \%$ & $93 \%$ \\
\hline
\end{tabular}

2. The data in each case represents the percentage coverage for each of the two coverage criteria. Each row describes the percentage coverage of the metamodel implementation (MM), the metrics implementation (Metrics) and the combined percentage coverage (All). Furthermore, each row represents cumulative coverage; for example, the line coverage value of $54 \%$ for group 2 includes the $51 \%$ line coverage achieved by group 1 . As can be seen from Table 4, the smaller test suites exhibit relatively poor coverage.

\subsection{Test oracle construction}

In this subsection we consider the construction of a reduced test suite that achieves the maximum coverage criteria possible for use as a test oracle for the metrics tool.

A number of techniques exist that can reduce test suites based on various constraints. For example, Harrold et al. outline techniques for test suite reduction and prioritisation based on coverage criteria [11]. However, since our test cases were being generated by Alloy roughly in order of size, a simpler approach was taken to test suite reduction:

1. As each test case is executed, the cumulative coverage of both criteria, is recorded.

2. Any test case that causes an increase in any one of the two coverage figures is added to the reduced suite.

3. This process is continued until either the maximum coverage has been achieved for both criteria or until all test cases have been examined.

In general this process will not perform as well as that of Harrold et al., but it is much simpler to implement. Applying this technique to the test cases, we generated a reduced test suite of 14 unique test cases. Table 5 lists the cumulative coverage data for each of these cases, labelled T1-T14. Three of these cases (T1-T3) originated from group 1, three (T4-T6) from group 2, and eight (T7-T14) from group 4. 
The 14 test cases almost achieved the maximum coverage possible. By inspecting the output from the Cobertura tool we were able to identify 10 lines of code that had not been covered by the reduced test suite. We then used Alloy to generate a valid metamodel instance to cover this situation. This model was added to our test suite and increased the coverage to the maximum value possible of $80 \%$ for code coverage and $93 \%$ for branch coverage.

The 15 test cases were then used to manually create a test oracle for the metrics tool. All 42 metrics were calculated by hand and recorded for each of the 15 test cases. We compared these values with the actual values computed by the metrics tool. In the next subsection, we briefly discuss the results of this along with the results from the smoke test.

\subsection{Discussion}

Using the above procedure we uncovered 6 bugs in the metrics tool. Four of these were detected by the smoke test and 2 with the test oracle. For example, for certain cohesion metrics (e.g. LCOM1), an auxiliary operation was specified in OCL to compute the set of method pairs in a Class. It was discovered that each method pair was being counted twice and thus returning a metric value outside of the expected bounds for the metrics. This error was corrected at the OCL level. Further, we identified and fixed the remaining bugs and regenerated the metrics tool.

In summary, we were able to partition the types of errors we found into three categories. The first category are bugs that are a result of the metric definitions themselves. For, example when a metric has no provision for a division by 0 . Second, are those introduced in the OCL where the definition has been incorrectly specified, for example a misplaced bracket in the OCL definition. Lastly, errors introduced by Octopus in transforming the UML/OCL to Java, for example incorrect casting of objects. Overall, our experience found this to be a relatively simple and effective way of increasing our confidence in the correctness of the automatically generated metrics tool.

\section{Related work}

The parallel between specification in Alloy and modelling in UML has been noted by Massoni et al. [13] and exploited by Anastasakis et al. [1]. Anastasakis et al. present a tool, UML2Alloy, that takes a UML class diagram, along with the associated OCL constraints, and translates this into an Alloy specification. The sample instances generated by the Alloy Analyser then correspond to object diagrams from the UML model. However, their tool does not provide any automated handling of the generated Alloy models.

Several other researchers have used Alloy to analyse and reason about metamodels. For instance, an alternative defi- nition of the UML metamodel is presented in [19] and analysed using Alloy. In [24], Alloy is used to formalise and analyse the package merge concept of the UML 2.0 metamodel. These approaches are similar to ours in that they use Alloy to describe a metamodel, as opposed to a model as with Anastasakis et al. However, the main focus of this research to date has been on the analysis of the UML metamodel. Our work, is concerned with using Alloy to analyse a metamodel for object-oriented software measurement. Moreover, these approaches have no automated support for metamodelling or for handling the generated models.

Some work related to ours is that of Gogolla et al. [10] who describe an approach to the automatic generation of model instances (snapshots) from UML class diagrams. ASSL (A Snapshot Sequence Language) is used to specify properties of a required model instance. Using their approach they generate two types of model instances, those that are test cases and those that are validation cases. The test cases confirm that models with certain properties can be created from the specification. The validation cases are used to show that certain properties of a model are a consequence of existing properties of the model. However, this approach is not fully automated as it requires the creation of scripts for each model in order to generate instances.

A related problem is that of generating metamodel instances for use in testing model transformations. Brottier et al. use an approach that determines the part of the metamodel that is relevant to the model transformation, and then determines coverage criteria based on this part of the metamodel [5]. This criteria is then used to generate metamodel instances. However, OCL constraints, an important part of a metamodel, cannot be directly reflected, leading to an under-specification of model instances.

Finally, an approach to metamodel instance generation is presented by Ehrig et al. [7]. This approach involves the automatic creation of an instance-generating graph grammar for the given metamodel. They also describe how to translate restricted OCL constraints to graph constraints. The grammar and the graph constraints are then used to create metamodel instances. However this approach does not support attribute values, only supports limited OCL constraints and cannot be used to verify properties of the metamodel.

\section{Concluding remarks}

In this paper we presented an approach to analysing MOF-compliant metamodels. We also presented a metamodel for coupling and cohesion measurement based on the work of Briand et al. and described how we used our approach to construct and analyse the metamodel. The metamodel and well-formedness rules were expressed in UML and OCL and a Java implementation and Alloy specification of the metamodel were generated by third-party tools. 
We used the Alloy specification to examine and validate the metamodel constraints, and to generate instantiations of the metamodel. We implemented a reflective instantiator to transform the automatically generated Alloy models into an instantiation of the Java implementation of the metamodel, generating a test suite for the metamodel-based metric calculation tool. Finally, we evaluated the adequacy of the test suite using several coverage criteria.

We identify the principal contributions of this paper as:

- The development and analysis of a MOF-compliant metamodel for coupling and cohesion metrics, based on the work of Briand et al., and the elimination of redundant constraints in that metamodel.

- The automation of the generation of metamodel instances from a UML/OCL specification that can be used as test data for metamodel-based software.

- A coverage-based analysis of the Alloy-generated test suite in terms of code coverage, thus "completing the circle" between lightweight formal methods and standard software testing techniques.

In future work, we plan to define a precise and complete scheme for transforming UML models and Java programs to instances of the metamodel presented in this paper.

Acknowledgements. Special thanks to Thomas Keane and the anonymous reviewers for their valuable comments on earlier revisions of this paper.

\section{References}

[1] K. Anastasakis, B. Bordbar, G. Georg, and I. Ray. UML2Alloy: A challenging model transformation. In Int. Conference on Model Driven Engineering Languages and Systems (MoDELS), volume 4735 of Lecture Notes in Computer Science, pages 436-450. Springer, 2007.

[2] A. L. Baroni, S. Braz, and F. B. e Abreu. Using OCL to formalize object-oriented design metrics definitions. In ECOOP Workshop on Quantative Approaches in ObjectOriented Software Engineering, Malaga, Spain, June 2002.

[3] L. Briand, J. Daly, and J. Wuest. A unified framework for cohesion measurement in object-oriented systems. Empirical Software Engineering, 3(1):65-117, 1998.

[4] L. Briand, J. Daly, and J. Wuest. A unified framework for coupling measurement in object-oriented systems. IEEE Transactions on Software Engineering, 25(1):91-121, 1999.

[5] E. Brottier, F. Fleurey, J. Steel, B. Baudry, and Y. L. Traon. Metamodel-based test generation for model transformations: an algorithm and a tool. In Intl. Symposium on Software Reliability Engineering, pages 85-94, Raleigh, NC, Nov. 2006.

[6] S. R. Chidamber and C. F. Kemerer. A metrics suite for object oriented design. IEEE Transactions on Software Engineering, 20(6):476-493, 1994.

[7] K. Ehrig, J. Küster, G. Taentzer, and J. Winkelmann. Generating instance models from meta models. In Intl. Conference on Formal Methods for Open Object-Based Distributed Systems, volume 4037 of Lecture Notes in Computer Science, pages 156-170. Springer, 2006.

[8] M. El-Wakil, A. El-Bastawisi, M. Riad, and A. Fahmy. A novel approach to formalize object-oriented design metrics. In Evaluation and Assessment in Software Engineering, Keele, UK, Apr. 2005.

[9] N. Fenton and S. Lawrence Pfleeger. Software Metrics: A Rigorous and Practical Approach. Intl. Thompson Computer Press, 1996.

[10] M. Gogolla, J. Bohling, and M. Richters. Validating UML and OCL models in USE by automatic snapshot generation. Journal on Software and System Modeling, 4(4):386-398, 2005.

[11] M. J. Harrold, R. Gupta, and M. L. Soffa. A methodology for controlling the size of a test suite. ACM Trans. Softw. Eng. Methodol., 2(3):270-285, 1993.

[12] D. Jackson. Software abstractions: logic, language, and analysis. The MIT Press, 2006.

[13] T. Massoni, R. Gheyi, and P. Borba. A UML class diagram analyzer. In 3rd International Workshop on Critical Systems Development with UML, Lisbon, Portugal, Oct. 2004.

[14] J. A. McQuillan and J. F. Power. Experiences of using the Dagstuhl Middle Metamodel for defining software metrics. In Intl. Conference on Principles and Practices of Programming in Java, pages 194-198, Germany, 2006.

[15] J. A. McQuillan and J. F. Power. Towards re-usable metric definitions at the meta-level. In PhD Workshop of the 20th European Conference on Object-Oriented Programming, Nantes, France, July 42006.

[16] J. A. McQuillan and J. F. Power. On the application of software metrics to UML models. In Models in Software Engineering, volume 4364 of Lecture Notes in Computer Science, pages 217-226. Springer, 2007.

[17] J. A. McQuillan and J. F. Power. Specifying coupling and cohesion metrics using OCL and Alloy. Technical Report NUIM-CS-TR-2008-01, Dept. of Computer Science, NUI Maynooth, Jan. 2008.

[18] T. Mens and M. Lanza. A graph-based metamodel for object-oriented software metrics. Electronic Notes in Theoretical Computer Science, 72(2), 2002.

[19] A. Naumenko and A. Wegmann. A metamodel for the Unified Modeling Language. In Proceedings of the 5th International Conference on The Unified Modeling Language, volume 2460, pages 2-17. Springer, 2002.

[20] Object Management Group. Meta Object Facility (MOF) Core Specification v2.0. Doc \# formal/06-01-01, Jan. 2006.

[21] Object Management Group. UML Superstructure Specification v2.1.1. Doc \# formal/07-02-05, Feb. 2007.

[22] J. Warmer and A. Kleppe. The Object Constraint Language: Getting your models ready for MDA. Addison-Wesley, 2003.

[23] F. G. Wilkie and T. J. Harmer. Tool support for measuring complexity in heterogeneous object-oriented software. In IEEE Intl. Conference on Software Maintenance, pages 152-161, Montréal, Canada, Oct. 2002.

[24] A. Zito and J. Dingel. Modeling UML2 package merge with Alloy. In First Alloy Workshop of the ACM SIGSOFT Symposium on Foundations of Software Engineering, Portland, OR, Nov. 2006. 